CIRJE-F-1040

\title{
Multi-Dimensional Pass-Through, Incidence, and the Welfare Burden of Taxation in Oligopoly
}

\author{
Takanori Adachi \\ Nagoya University \\ Michal Fabinger \\ University of Tokyo
}

February 2017

CIRJE Discussion Papers can be downloaded without charge from:

http://www.cirje.e.u-tokyo.ac.jp/research/03research02dp.html

Discussion Papers are a series of manuscripts in their draft form. They are not intended for circulation or distribution except as indicated by the author. For that reason Discussion Papers may not be reproduced or distributed without the written consent of the author. 


\title{
Multi-Dimensional Pass-Through, Incidence, and the Welfare Burden of Taxation in Oligopoly*
}

\author{
TAKANORI AdACHI ${ }^{\dagger} \quad$ Michal FABINGER ${ }^{\ddagger}$
}

February 18, 2017

\begin{abstract}
This paper studies welfare consequences of unit and ad valorem taxes in oligopoly with general demand, non-constant marginal costs, and a generalized type of competition. We present formulas providing connections between marginal cost of public funds, tax incidence, unit tax pass-through, ad valorem tax pass-through, and other economic quantities of interest. We show that there exists a simple, empirically relevant set of sufficient statistics for the marginal cost of public funds, namely the pass-through and the industry demand elasticity. Specializing to the case of price or quantity competition, we show how marginal cost of public funds and pass-through may be expressed using elasticities and curvatures of demand and inverse demand. These results apply also to symmetric oligopoly with multi-product firms. Finally, we present a generalization with the tax revenue function specified as a general function parametrized by a vector of tax parameters. We define multidimensional generalizations of pass-through and show that they are crucial for evaluating welfare changes in response to changes in taxation.
\end{abstract}

Keywords: Oligopoly; Unit tax; Ad valorem tax; Welfare; Pass-through; Elasticities; Curvatures; Sufficient statistics.

JEL classification numbers: D43; L11; L13.

*Adachi and Fabinger acknowledge a Grant-in-Aid for Scientific Research (C) (15K03425) and a Grant-in-Aid for Young Scientists (A) (26705003) from the Japan Society for the Promotion of Science, respectively. Adachi also acknowledges financial support from the Japan Economic Research Foundation.

${ }^{\dagger}$ School of Economics, Nagoya University, 1 Furo-cho, Chikusa-ku, Nagoya 464-8601, Japan. E-mail: adachi.t@soec.nagoya-u.ac.jp

${ }^{\ddagger}$ Graduate School of Economics, University of Tokyo, 7-3-1 Hongo, Bunkyo-ku, Tokyo 113-0033, Japan. E-mail: fabinger@e.u-tokyo.ac.jp. Fabinger is also a research associate at CERGE-EI, Prague, the Czech Republic. 


\section{Introduction}

The marginal cost of public funds, i.e. the marginal social welfare loss associated with raising additional tax revenue, is a crucial characteristic that a policymaker needs to take into account when designing an optimal system of taxes. ${ }^{1}$ However, it is somewhat surprising that relatively little is known about the general mechanism of how tax levies are passed on to final prices as well as their welfare consequences in oligopoly, a ubiquitous feature of competition in the real-world economy.

In this paper, we aim to contribute to the general understanding of the welfare consequences of taxation in symmetric oligopolistic markets with a general (first-order) type of competition (of single-product or multi-product firms), with a possibly non-constant marginal cost, and with non-zero unit and ad valorem tax rates. Specifically, we establish connections between welfare measures, namely the marginal cost of public funds for unit taxes and ad valorem taxes, and variables that are easily interpretable from an empirical standpoint, namely the pass-through of these taxes (i.e. the marginal change of prices induced by tax rate changes). ${ }^{2}$ In particular, we show that with a general type of competition, there exists a simple set of sufficient statistics that determines the marginal cost of public funds of unit and ad valorem taxes, namely pass-through of these taxes and the industry demand elasticity (in addition to the easily observed taxation levels). ${ }^{3}$

\footnotetext{
${ }^{1}$ In the absence of other considerations, the marginal cost of public funds should be equalized across markets in order to maximize social welfare.

${ }^{2}$ The usefulness of pass-through in welfare analysis has been verified by related studies such as Cowan (2012); Miller, Remer, and Sheu (2013); Weyl and Fabinger (2013); Gaudin and White (2014); MacKay, Miller, Remer, and Sheu (2014); Adachi and Ebina (2014a,b); Chen and Schwartz (2015); Gaudin (2016); Cowan (2016); and Alexandrov and Bedre-Defolie (2017). See also Ritz (2017) for an excellent survey of theoretical studies on pass-through and pricing under imperfect competition.

${ }^{3}$ The sufficient-statistics approach to connecting structural and reduced-form methods, as advocated by Chetty (2009), has been successful in empirical economics. For example, in the study by Atkin and Donaldson (2016), the pass-through rate provides a sufficient statistic for welfare implications of intra-national trade costs in low-income countries, without the need for a full demand estimation. Similarly, Ganapati, Shapiro, and Walker (2017) examine the welfare effects of input taxation, where a unit tax is levied on the input. These effects are related to the effects of unit taxes
} 
This result is a part of a larger set of relationships that link economic quantities of interest. We derive succinct formulas that relate the marginal cost of public funds to pass-through of taxes of the same type. We also establish a relationship that connects pass-through of unit taxes and pass-through of ad-valorem taxes in the same market. Further, we derive convenient expressions for values of unit and ad valorem pass-through that are valid under a general type competition and have not appeared in the previous literature.

In addition, specializing to price (differentiated Bertrand) competition and quantity (differentiated Cournot) competition, we show how marginal cost of public funds and pass-through may be expressed using elasticities and curvatures of demand and inverse demand, and provide illustrative examples. Our results apply without change also to fully symmetric oligopoly with multi-product firms. Throughout the analysis, we allow for finite levels of unit and ad valorem taxes. However, we also discuss some additional simplifications that appear when instead the initial level of taxes is zero.

Finally, we generalize our results to a significantly more general specification of taxation that involves multiple tax parameters. We define two different types of pass-through vectors: the pass-through rate vector and the pass-through quasielasticity vector. We study their properties and show that they are crucial for evaluating welfare changes in response to changes in taxation. Special cases involve not only unit and ad valorem taxation, but also exogenous competition discussed by Weyl and Fabinger (2013), as well as, for example, value-added tax, under which the firm can deduct a portion of its costs from its profit for taxation purposes.

From both theoretical and empirical standpoints, it is desirable to be able to understand the welfare properties of oligopolistic markets with a general type of (first-order) competition. In real-world situations, the behavior of firms corresponds

on output, but not identical. See also Fabra and Reguant (2014); Shrestha and Markowitz (2016); Duso and Szücs (2016); Stolper (2016); and Hong and Li (2017) for studies with the same spirit. In contrast, structural studies with a full specification of demands include Kim and Cotterill (2008); Bonnet, Dubois, Villas-Boas, and Klapper (2013); Bonnet and Réquillart (2013); Campos-Vázquez and Medina-Cortina (2015); Griffith, Nesheim, and O'Connell (2015); Miller, Remer, Ryan, and Sheu (2016); and Miller, Osborne, and Sheu (2017). 
to neither the idealized price competition nor the idealized quantity competition. Price competition does not allow for any friction in scaling production levels up or down, yet in reality there tend to be substantial frictions, such as those related to financial constraints or the labor market. Quantity competition implies that the firm will not be able to increase production levels when its competitor suddenly decides to increase prices. In reality, such adjustment is feasible, since capacity utilization is typically less than complete, and even if the firm is operating at full capacity, boosting production levels is possible by overtime work or by hiring temporary workers. Moreover, firms may behave to some extent in a collusive way. Although the realities of firm competition may be complicated, it is possible to capture their essence by working with a general type of competition, using the conduct index. ${ }^{4}$

Besides working with a general type of competition, it is also useful to relax the assumption of constant marginal costs that often appears in the literature. Production technologies often have non-trivial structure, and so does the internal organization of the firm. For example, if a firm decides to operate at a larger scale, it may take advantage of technological and logistical economies of scale, but at the same time, it may face more severe principal-agent problems as top managers have to delegate responsibilities to lower-level managers. The interplay between these forces can lead to a non-trivial dependence of the marginal cost of production on the scale of the operation.

This paper is related to the inspiring study by Häkner and Herzing (2016), which motivates parts of this work. In the special case of linear demand, and constant marginal cost, Häkner and Herzing (2016, p.147) explain that as long as the initial level of taxes is zero, the marginal cost of public funds for unit taxation equals $M C_{t}=\theta \rho_{t}$, where $\rho_{t}$ is the pass-through rate (the marginal effect of taxes on prices), and $\theta$, usually referred to as the conduct parameter, measures the degree of non-competitiveness of the market (for example, in the case of monopoly $\theta=1$, while under perfect competition $\theta=0$ ). For ad valorem taxes, Häkner and Herzing

\footnotetext{
${ }^{4}$ For details of this approach, see Bresnahan (1989) and Weyl and Fabinger (2013). It has been successfully applied also to more general situations, such as selection markets (Mahoney and Weyl (2017)) or supply chains (Gaudin (2016)).
} 
(2016) provide a similar formula. They show, however, that if we let the initial level of taxes be non-zero, those formulas are no longer valid. For this reason, they are forced to analyze the magnitude of the marginal cost of public funds on a case-bycase basis using explicit solutions to specific models.

This situation represents a puzzle. If there are simple formulas for the marginal cost of public funds that were valid at zero taxes, is there no compact generalization of these expressions in the case of non-zero taxes? If there is no such generalization, that would be an obstacle to empirical work, since we would have to make additional modeling assumptions before obtaining empirical estimates of the marginal cost of public funds. Our paper provides a solution to this problem. In particular, Propositions 1 and 2 present formulas for the marginal cost of public funds that are valid even when the initial level of (ad valorem and unit) taxes is non-zero. They are a bit longer than $M C_{t}=\theta \rho_{t}$, but still very manageable. They also represent a starting point for the topics discussed in the rest of the paper. These results with a non-zero initial taxes being allowed, which are differentiated from Weyl and Fabinger (2013) and Häckner and Herzing (2016), should be useful if one needs to evaluate the marginal cost of taxation when some tax has been already implemented.

The welfare cost of taxation has been extensively studied at least since Pigou (1928). The majority of the studies simply assume perfect competition. ${ }^{5}$ As is widely known, under perfect competition, unit tax and ad valorem tax are equivalent, and whether consumers or producers bear more is determined by the relative elasticities of demand and supply. The initial attempt to relax the assumption of perfect competition started with an analysis of homogeneous-product oligopoly under quantity competition, i.e., Cournot oligopoly. Notably, Delipalla and Keen (1992), Skeath and Trandel (1994), and Hamilton (1999)) compare ad valorem and unit taxes in such a setting. Then, Anderson, de Palma, and Kreider (2001a) extend these results importantly to the case of differentiated oligopoly under price competition. In particular, Anderson, de Palma, and Kreider (2001a) find that whether the after-tax

\footnotetext{
${ }^{5}$ See, e.g., Vickrey (1963), Buchanan and Tullock (1965), Johnson and Pauly (1969), and Browing (1976) for early studies. A study of unit and ad valorem taxation under imperfect competition with homogenous products dates back to Delipalla and Keen (1992).
} 
price for firms and their profits rise by a change in ad valorem tax depends importantly on the ratio of the curvature of the firm's own demand $\left(\varepsilon_{m}\right.$ in their notation, and $\alpha_{F}$ in our notation below) to the elasticity of the market demand $\varepsilon_{D D}$ in their notation, and $\epsilon$ in our notation).

We extend Anderson, de Palma, and Kreider's (2001a) setting and results in a number of important directions. First, we consider the general mode of competition, captured by the conduct index, including both quantity and price competition. Second, we provide a complete characterization of tax burdens that enables one to quantitatively compare consumers' burden with producers' burden, whereas Anderson, de Palma, and Kreider's (2001a) focus only on the effective prices for consumers and producers' profits. Third, while Anderson, de Palma, and Kreider's (2001a) assume constant marginal cost, we allow non-constant marginal cost and show how this generalization makes a difference in our general formulas. Fourth, we further generalize the initial tax level. When they analyze the effects of a unit tax, Anderson, de Palma, and Kreicher (2001a) assume that ad valorem tax is zero, and vice versa. In contrast, we allow non-zero initial taxes in both dimensions. Finally, and importantly, we generalize these results to the case of a very general type of taxation. This opens up the possibility to study a wide range of interventions/taxes and to derive convenient sufficient statistics for characteristics, including welfare characteristics, of the markets of interest.

In the next section, we study the problem of oligopoly with a general type of competition. In Section 3, we specialize to the case of price or quantity competition. Section 4 explains how our results apply to the case of oligopoly with multi-product firms. Section 5 generalizes the results from unit and ad-valorem taxation to much more flexible taxation parameterized by $d$ different tax parameters and discusses the implications of these general results. Section 6 concludes. 


\section{Taxation and Welfare in Symmetric Oligopoly}

We study oligopolistic markets with $n$ symmetric firms and a general (first-order) mode of competition and the resulting symmetric equilibria. ${ }^{6}$ Our discussion applies to single-product firms as well as to multi-product firms if intra-firm symmetry conditions are satisfied, as discussed in Section 4. For simplicity of exposition, we use terminology corresponding to single-product firms here, and later we discuss how to interpret the results in the case of multi-product firms.

The demand for firm $j$ 's product $q_{j}=q_{j}\left(p_{1}, \ldots, p_{n}\right) \equiv q_{j}(\mathbf{p})$ depends on the vector of prices $\mathbf{p} \equiv\left(p_{1}, \ldots, p_{n}\right)$ charged by the individual firms. The demand system is symmetric and the cost function $c\left(q_{j}\right)$ is the same for all firms. We assume that $q_{j}(\cdot)$ and $c(\cdot)$ are twice differentiable and conditions for the uniqueness of equilibrium and the associated second-order conditions are satisfied.

We denote by $q(p)$ the per-firm industry demand corresponding to symmetric prices: $q(p) \equiv q_{j}(p, \ldots, p)$. The elasticity of this function, defined as $\epsilon(p) \equiv$ $-p q^{\prime}(p) / q(p)>0$ and referred to as the price elasticity of industry demand, should not be confused with the elasticity of the residual demand that any of the firms faces. ${ }^{7}$ We also use the notation $\eta(q)=1 /\left.\epsilon(p)\right|_{q(p)=q}$ for the reciprocal of this elasticity as a function of $q$. For the corresponding functional values, when we do not need to specify explicitly their dependence on either $q$ or $p$, we use $\eta$ interchangeably with $1 / \epsilon$.

We introduce two types of taxation: a unit tax $t$ and an ad valorem tax $v$, with firm $j$ 's profit being $\pi_{j}=(1-v) p_{j}\left(q_{j}\right) q_{j}-t q_{j}-c\left(q_{j}\right)$. At symmetric quantities the government tax revenue per firm is $R(q) \equiv t q+v p(q) q$, and we denote by $\tau(q)$ the fraction of firm's pre-tax revenue that is collected by the government in the form of

\footnotetext{
${ }^{6}$ Although for brevity we speak of a general mode of competition, we consider only "first-order" competition, in the sense of the firms making decisions based on marginal cost and marginal revenue. This excludes, for example, the possibility of each producer being composed of two vertically related firms where the upstream firm sets prices for a relationship-specific intermediate good, as in the usual double-marginalization problem specification.

${ }^{7}$ The elasticity $\epsilon$ here corresponds to $\epsilon_{D}$ in Weyl and Fabinger (2013, p. 542). Note that $q^{\prime}(p)=$ $\partial q_{j}(\mathbf{p}) / \partial p_{j}+(n-1) \partial q_{j}(\mathbf{p}) /\left.\partial p_{j^{\prime}}\right|_{\mathbf{p}=(p, \ldots, p)}$ for any two distinct indices $j$ and $j^{\prime}$. We will define the firm's elasticity and other related concepts in Section 3.
} 
taxes: $\tau(q) \equiv R(q) / q=v+t / p(q)$. We define the conduct index $\theta(q)$ (or conduct parameter) as

$$
\theta(q)=\frac{1}{\eta(q) p(q)}\left(p(q)-\frac{t+m c(q)}{1-v}\right),
$$

where $m c(q) \equiv c^{\prime}(q)$ is the marginal cost of production, and we denote by $\theta$ its functional value at the equilibrium quantity. ${ }^{8,9}$ The marginal welfare cost $M C_{t}$ or $M C_{v}$ of raising government revenue by the unit tax $t$ or the ad valorem tax $v$, i.e. the marginal cost of public funds associated with such tax, are defined as

$$
M C_{t} \equiv-\left(\frac{\partial W}{\partial t}\right)^{-1} \frac{\partial R}{\partial t}, \quad M C_{v} \equiv-\left(\frac{\partial W}{\partial v}\right)^{-1} \frac{\partial R}{\partial v},
$$

where $W$ is the social welfare per firm, which includes consumer surplus, producer surplus, and government tax revenue. We define the unit tax pass-through rate $\rho_{t}$ and the ad valorem tax pass-through semi-elasticity $\rho_{v}$ as: ${ }^{10}$

$$
\rho_{t}=\frac{\partial p}{\partial t}, \quad \rho_{v}=\frac{1}{p} \frac{\partial p}{\partial v}
$$

Consider an infinitesimal change in the unit tax, with the initial tax level $(t, v)$. As mentioned in the introduction, in the special case of zero initial taxes, linear demand, and constant marginal cost, Häckner and Herzing (2016, p. 147) show that $M C_{t}=\theta \rho_{t}$. They further show that at non-zero initial taxes the formula no longer applies. In the absence of such formula, they were forced to study the marginal cost of public funds on a case-by-case basis, for different specifications of demand and cost.

We aim to find a generalization of the formula $M C_{t}=\theta \rho_{t}$ that would be applicable even at non-zero initial taxes. In this situation, the expression $\theta \rho_{t}$ as a measure of marginal cost of public funds has two deficiencies. First, the expression is simply proportional to $\theta$, but when $v$ is large, the firms sell at prices that are too high from the social perspective not because of a lack of competitiveness, but

\footnotetext{
${ }^{8}$ The term "conduct parameter" originates from situations where it was supposed to be constant. Since here we allow it to be variable, we opt for the term "conduct index".

${ }^{9}$ More precisely, $\theta(q)$ is defined to be a function independent of the cost side of the economic problem such that the symmetric equilibrium condition may be written in the form of Equation 1.

${ }^{10}$ Note that Häckner and Herzing (2016) use the symbol $\rho_{v}$ for the ad-valorem tax pass-through rate $\partial p / \partial v$, which would be $p \rho_{v}$ in our notation.
} 
because the tax effectively raises their perceived cost. When $v$ is large, we would expect the marginal cost of public funds to be less sensitive to $\theta$, for a given value of $\rho_{t}$. Second, the expression $\theta \rho_{t}$ does not explicitly feature the level of the unit tax $t$. However, a situation where $t$ is large and $m c$ small is very different from a situation where $t$ is small and $m c$ large, even if the equilibrium prices and quantities are the same. In the former case, raising additional tax revenue is quite harmful, since firms' production cuts will not substantially decrease the total technological (i.e., pre-tax) cost of production. In the latter case, raising additional tax revenue is less harmful since it leads to reduced total technological cost. Based on this intuition, we would expect the marginal cost of public funds to be an increasing function of $t .^{11}$

It turns out that it is possible to identify a formula with precisely these properties, as the following proposition shows.

Proposition 1. Marginal cost of public funds for unit taxation. Under symmetric oligopoly with a possibly non-constant marginal cost, the marginal cost of public funds associated with a unit tax may be expressed as

$$
M C_{t}=\frac{(1-v) \theta+\epsilon \tau}{\frac{1}{\rho_{t}}+v-\epsilon \tau} .
$$

Proof. Using Equation 1 to substitute for $m c$, we first obtain a useful expression for the markup: $p-m c=t+p v+p(1-v) \eta \theta$. Now consider an infinitesimal change $d t$ in the unit tax that induces a change $d p$ in the equilibrium price and a change $d q$ in the equilibrium quantity. These are related by $d t=d p / \rho_{t}=-\eta p d q /\left(q \rho_{t}\right)$. The corresponding change in social welfare per firm is $d W=(p-m c) d q=t d q+$ $v p d q+(1-v) p \eta \theta d q$, and the change in tax revenue per firm is $d R=(t+v p) d q+$ $v q d p+q d t=(t+v p) d q-v p \eta d q-\eta p d q / \rho_{t}$. Combining these relationships gives the result

$$
M C_{t}=-\frac{d W}{d R}=-\frac{t+v p+(1-v) p \eta \theta}{t+v p-v p \eta-\frac{1}{\rho_{t}} p \eta}=\frac{(1-v) \eta \theta+\frac{t}{p}+v}{\frac{1}{\rho_{t}} \eta+v \eta-\frac{t}{p}-v}=\frac{(1-v) \theta+\epsilon \tau}{\frac{1}{\rho_{t}}+v-\epsilon \tau} .
$$

\footnotetext{
${ }^{11}$ In the sense of making the change $t \rightarrow t+\Delta t$, and simultaneously $c(q) \rightarrow c(q)-q \Delta t$ in order to keep $q, \theta$, and $\rho_{t}$ at some fixed values.
} 

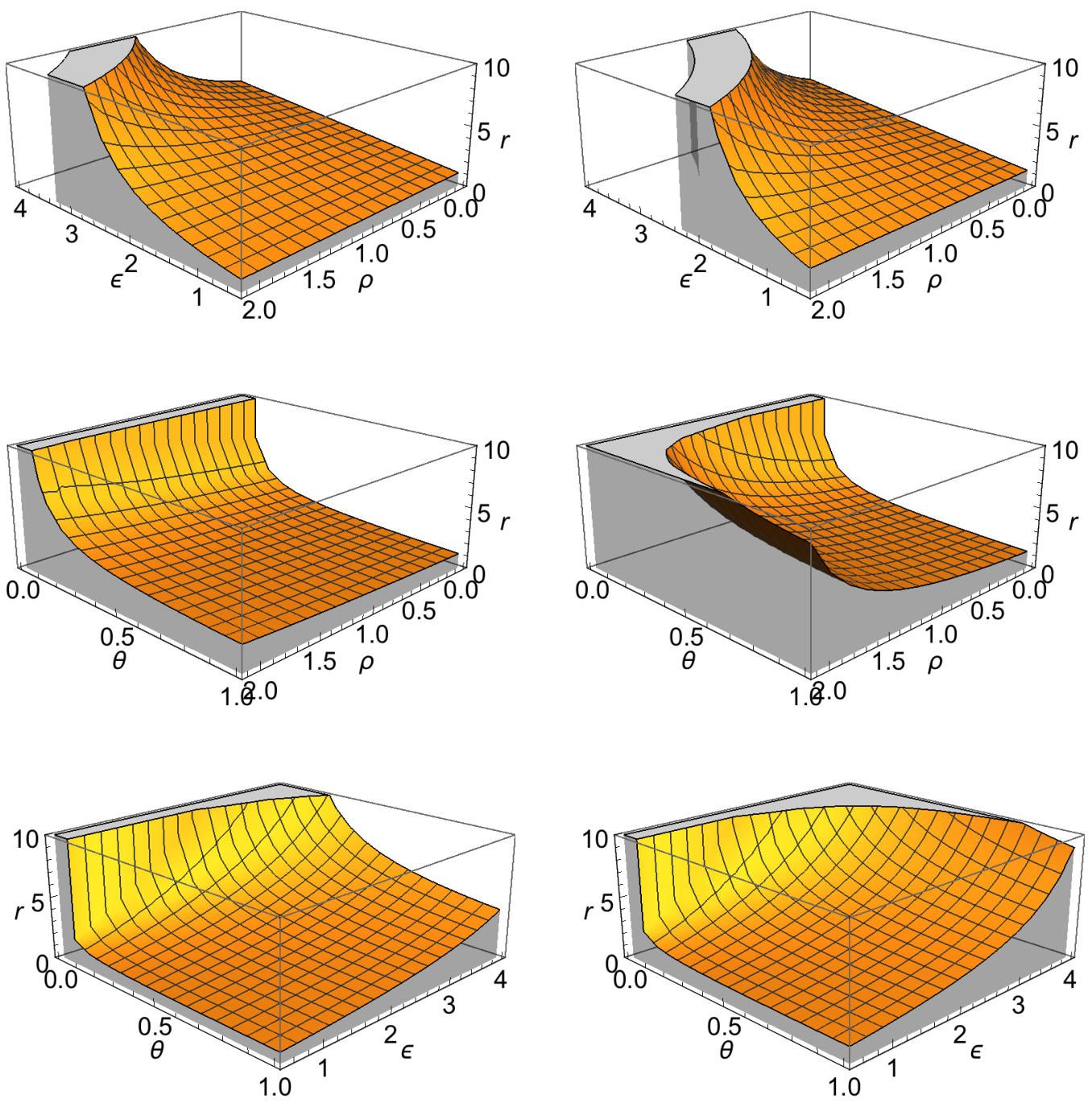

Figure 1: The ratio of the actual marginal cost of public funds $M C$ and the naive expression $\theta \rho$ discussed on p. 8 , plotted as a function of combinations of the conduct index $\theta$, the pass-through $\rho$, and the industry demand elasticity $\epsilon$. The figures on the left correspond to infinitesimal changes in unit taxation: $\rho$ stands for $\rho_{t}$ and $M C$ stands for $M C_{t}$. The numerical values were chosen to be $t=0, v=0.2, \tau=0.2$. The figures on the right correspond to infinitesimal changes in ad valorem taxation: $\rho$ stands for $\rho_{v}$ and $M C$ stands for $M C_{v}$. The numerical values were chosen to be $t / p=0.2, v=0, \tau=0.2$. The top figures correspond to $\theta=0.3$, the middle figures correspond to $\epsilon=2$, and the bottom figures correspond to $\rho=1$. 
For the welfare effects of an infinitesimal change in ad valorem tax we obtain the following proposition.

Proposition 2. Marginal cost of public funds for ad valorem taxation. Under symmetric oligopoly with a possibly non-constant marginal cost, the marginal cost of public funds associated with an ad valorem tax may be expressed as

$$
M C_{v}=\frac{(1-v) \theta+\epsilon \tau}{\frac{1}{\rho_{v}}+v-\epsilon \tau} .
$$

Proof. In analogy with the previous proof, consider an infinitesimal change $d v$ in the ad valorem tax that induces a change $d p$ in the equilibrium price and a change $d q$ in the equilibrium quantity, related by $d v=d p /\left(p \rho_{v}\right)=-\eta d q /\left(q \rho_{v}\right)$. The change in social welfare per firm is again $d W=(p-m c) d q=t d q+v p d q+(1-v) p \eta \theta d q$. The change in tax revenue per firm can be written as $(t+v p) d q+v q d p+p q d v=$ $(t+v p) d q-v p \eta d q-p \eta d q / \rho_{v}$. Combining these relationships leads to the result

$$
M C_{t}=-\frac{d W}{d R}=-\frac{t+v p+(1-v) p \eta \theta}{t+v p-v p \eta-\frac{1}{\rho_{v}} p \eta}=\frac{(1-v) \eta \theta+\frac{t}{p}+v}{\frac{1}{\rho_{v}} \eta+v \eta-\frac{t}{p}-v}=\frac{(1-v) \theta+\epsilon \tau}{\frac{1}{\rho_{v}}+v-\epsilon \tau} .
$$

Figure 1 documents that these expressions for the marginal cost of public funds $M C_{t}$ and $M C_{v}$ evaluated at realistic values of taxes and other economic variables are very different from the values of the expressions $\theta \rho_{t}$ and $\theta \rho_{v}$ (discussed on p. 8) that would be equal to $M C_{t}$ and $M C_{v}$ if taxes were zero.

For the incidence of taxation at non-zero unit and ad valorem taxes, we obtain similarly succinct formulas. We define the incidence $I_{t}$ of unit taxation as the ratio of changes $d C S$ in (per-firm) consumer surplus and changes $d P S$ in (per-firm) producer surplus induced by an infinitesimal increase $d t$ in the unit tax $t$. The incidence $I_{v}$ is defined analogously.

Proposition 3. Incidence of taxation. Under symmetric oligopoly with a general type of competition and with a possibly non-constant marginal cost, the incidence of unit taxes $I_{t}$ and of ad valorem taxes $I_{v}$ is given by

$$
\frac{1}{I_{t}}=\frac{1}{\rho_{t}}-(1-v)(1-\theta)
$$




$$
\frac{1}{I_{v}}=\frac{1}{\rho_{v}}-(1-v)(1-\theta)
$$

Proof. The impact of a change $d t$ in the tax $t$ on consumer surplus (per firm) is $d C S=-q d p=-q \rho_{t} d t$. The impact on producer surplus is

$$
\begin{gathered}
d P S=d((1-v) p q-c(q)-t q)=-q d t+(1-v) p d q+(1-v) q d p-m c d q-t d q, \\
d P S=-q d t+(1-v) q \rho_{t} d t+((1-v) p-m c-t) d q .
\end{gathered}
$$

Substituting for $m c$ from Equation 1 as $m c=(1-v)(1-\eta \theta) p-t$ gives

$$
\begin{gathered}
d P S=-q d t+(1-v) q \rho_{t} d t+(1-v) \eta \theta p d q=-q d t+(1-v) q \rho_{t} d t-(1-v) \theta q d p \\
d P S=-q d t+(1-v) q \rho_{t} d t-(1-v) \theta q \rho_{t} d t=\left((1-v)(1-\theta) \rho_{t}-1\right) q d t .
\end{gathered}
$$

The reciprocal of the incidence ratio is

$$
\frac{1}{I_{t}}=\frac{d P S}{d C S}=\frac{(1-v)(1-\theta) q \rho_{t}-q}{-q \rho_{t}}=\frac{1}{\rho_{t}}-(1-v)(1-\theta) .
$$

Similarly, for infinitesimal changes in ad valorem taxes we proceed analogously. The change in consumer suprlus is $d C S=-q d p=-q p \rho_{v} d v$. For the change in producer surplus we have

$d P S=d((1-v) p q-c(q)-t q)=-p q d v+(1-v) p d q+(1-v) q d p-m c d q-t d q$.

Manipulating the last four terms on the right-hand side in the same way as before leads to

$$
\begin{gathered}
d P S=-p q d v+(1-v) p d q+(1-v) q d p-m c d q-t d q \\
d P S=-p q d v+(1-v) q p \rho_{v} d v-(1-v) \theta q p \rho_{v} d v=\left((1-v)(1-\theta) \rho_{v}-1\right) q p d v .
\end{gathered}
$$

The reciprocal of the incidence ratio then becomes

$$
\frac{1}{I_{t}}=\frac{d P S}{d C S}=\frac{(1-v)(1-\theta) \rho_{v} q-q}{-q \rho_{v}}=\frac{1}{\rho_{v}}-(1-v)(1-\theta)
$$

In the case of zero ad valorem tax, the expression for $I_{t}$ reduces to the one in Weyl and Fabinger (2013). Next, we show how $\rho_{t}$ and $\rho_{v}$ are related in the following proposition. 


\section{Proposition 4. Relationship between pass-through of ad valorem and unit}

taxes. Under symmetric oligopoly with a possibly non-constant marginal cost, the pass-through semi-elasticity $\rho_{v}$ of an ad valorem tax may be expressed in terms of the unit tax pass-through rate $\rho_{t}$, the conduct index $\theta$, and the industry demand elasticity $\epsilon$ as

$$
\rho_{v}=\frac{\epsilon-\theta}{\epsilon} \rho_{t}
$$

Proof. Let us consider a simultaneous infinitesimal change $d t$ and $d v$ in the taxes $t$ and $v$ that leaves the equilibrium price (and quantity) unchanged, which requires the effective marginal cost $(t+m c) /(1-v)$ in Equation 1 to remain the same. This implies the comparative statics relationship

$$
d t \frac{\partial}{\partial t} \frac{t+m c}{1-v}+d v \frac{\partial}{\partial v} \frac{t+m c}{1-v}=0 \Rightarrow \frac{d t}{1-v}+\frac{t+m c}{(1-v)^{2}} d v=0 \Rightarrow d t=-\frac{t+m c}{1-v} d v .
$$

Note that here we do not need to take derivatives of $m c$ even though it depends on $q$, simply because by assumption the quantity is unchanged. The total induced change in price, which generally would be expressed as $d p=\rho_{t} d t+\rho_{v} p d v$, must equal zero in this case, implying the result

$\rho_{t} d t+\rho_{v} p d v=0 \Rightarrow-\frac{t+m c}{1-v} \rho_{t} d v+\rho_{v} p d v=0 \Rightarrow \rho_{v}=(1-\eta \theta) \rho_{t} \Rightarrow \rho_{v}=\frac{\epsilon-\theta}{\epsilon} \rho_{t}$.

The results above allow us to construct a convenient set of sufficient statistics for the marginal cost of public funds.

Proposition 5. Sufficient statistics for marginal costs of public funds.

Under symmetric oligopoly with a possibly non-constant marginal cost, the unit passthrough rate $\rho_{t}$, the ad valorem pass-through semi-elasticity $\rho_{v}$, and the elasticity $\epsilon$ of industry demand (together with the tax rates and the fraction $\tau$ of the firm's pre-tax revenue collected by the government in the form of taxes) serve as sufficient statistics for the marginal cost of public funds both with respect to unit taxes and ad valorem taxes. In particular:

$$
M C_{t}=\frac{(1-v+\tau) \rho_{t}-(1-v) \rho_{v}}{1+(v-\epsilon \tau) \rho_{t}} \epsilon
$$




$$
M C_{v}=\frac{(1-v+\tau) \rho_{t}-(1-v) \rho_{v}}{1+(v-\epsilon \tau) \rho_{v}} \frac{\epsilon \rho_{v}}{\rho_{t}}
$$

Proof. Proposition 4 allows us to express the conduct index $\theta$ as $\theta=\left(1-\rho_{v} / \rho_{t}\right) \epsilon$. Substituting this into the relationships in the first two propositions gives the desired result.

Note that to use these formulas, we do not need to make a guess about the degree of competitiveness based on the number of firms. For example, in the case of the assumption of Cournot competition, researchers often may observe the number $n$ of firms and conclude that the value of conduct index is $\theta=1 / n$. The general formulas in the last proposition do no rely on such assumptions; the selected sufficient statistics allow us to empirically estimate $\theta$, i. e. allow us to measure the degree of cartelization of those $n$ firms.

Proposition 6. Pass-through under general symmetric oligopoly. Under symmetric oligopoly with a general mode of competition and with a possibly nonconstant marginal cost:

$$
\rho_{t}=\frac{1}{1-v} \frac{1}{1-(\eta+\chi) \theta+\epsilon q(\theta \eta)^{\prime}+\frac{1-\tau}{1-v} \epsilon \chi},
$$

where the derivative is taken with respect to $q$ and $\chi \equiv m c^{\prime} q / m c$. Further,

$$
\rho_{v}=\frac{\epsilon-\theta}{(1-v) \epsilon} \frac{1}{1-(\eta+\chi) \theta+\epsilon q(\theta \eta)^{\prime}+\frac{1-\tau}{1-v} \epsilon \chi} .
$$

Proof. Consider the comparative statics with respect to a small change $d t$ in the perunit tax $t$. Totally differentiating the equation $p-\theta m s=(m c+t) /(1-v)$ gives the comparative statics equation $d p-d(\theta m s)=(d m c+d t) /(1-v)$. Further, denoting by prime the derivatives with respect to quantity, we have $d(\theta m s)=(\theta m s)^{\prime} d q=$ $-q \epsilon(\theta m s)^{\prime} d p / p=-q \epsilon(\theta \eta p)^{\prime} d p / p=-q \epsilon(\theta \eta)^{\prime} d p-\frac{q}{p} \theta p^{\prime} d p=\left(\theta \eta-q \epsilon(\theta \eta)^{\prime}\right) d p$. The change in marginal cost can be written in terms of the marginal cost elasticity as $d m c=\chi m c d q / q=-\chi \epsilon m c d p / p$. In this expression, the marginal cost may be eliminated using $p-\theta m s=(m c+t) /(1-v) \Rightarrow m c=(1+v)\left(p-\theta q p^{\prime}\right)-t=$ $(1-v)(1+\theta \eta) p-t$, which leads to $d m c=-((1-v)(1+\theta \eta)-t / p) \chi \epsilon d p$. In terms 
of the per-unit-revenue tax burden $\tau \equiv v+t / p$, this is $d m c=-((1-v)(1+\theta \eta)-$ $\tau+v) \chi \epsilon d p=-((1-v) \theta \eta+1-\tau) \chi \epsilon d p$. Substituting the resulting expressions for $d(\theta m s)$ and $d m c$ into the comparative statics equation gives

$$
\rho_{t}=\frac{d p}{(1-v)(d p-d(\theta m s))-d m c}=\frac{1}{(1-v)\left(1-\theta \eta+(\theta \eta)^{\prime} \epsilon q\right)+(1-v) \theta \chi+(1-\tau) \epsilon \chi},
$$

which is equivalent to the expression for $\rho_{t}$ in the proposition. The expression for $\rho_{v}$ then follows by Proposition 4 .

Let us also point out that the exchange rate pass-through can be included naturally in our framework. ${ }^{12}$ Suppose that domestic firms in a country of interest use some imported inputs for production. For concreteness, let us specify the profit function of firm $j$ as $\pi_{j}=\left[(1-v) p_{j}-t\right] q_{j}-(1+a e) c\left(q_{j}\right)$, where the constant coefficient $a$ measures the importance imported inputs and $e>0$ is the exchange rate. Notice that the firm's profit is rewritten as $\pi_{j}=(1+a e)\left[\left(\frac{1-v}{1+a e} p_{j}-\frac{t}{1+a e}\right) q_{j}-c\left(q_{j}\right)\right]$. Since the first factor on the right-hand side is constant, the firm will behave as if its profit function was simply $\tilde{\pi}_{j}=\left[(1-\tilde{v}) p_{j}-\tilde{t}\right] q_{j}-c\left(q_{j}\right)$, with $\tilde{v} \equiv(v+a e) /(1+a e)$ and $\tilde{t} \equiv t /(1+a e)$. By utilizing the explicit expressions for the derivatives $\partial \tilde{v} / \partial e=$ $(a-v) /(1+a e)^{2}$ and $\partial \tilde{t} / \partial e=-a t /(1+a e)^{2}$, one can analyze the effect of a change in the exchange rate $e$ on social welfare. Note that this is simply interpreted as the cost pass-through as well (see the references in Footnote 12 for empirical studies).

\section{Taxation and Welfare under Specific Types of Competition}

In this section we show that for price competition and quantity competition in differentiated oligopoly, our general expressions of the marginal cost of public funds and pass-through lead to expressions in terms of demand primitives such as the elasticities and the curvatures, and the marginal most elasticity $\chi$ defined above. We also provide parametric examples for these results.

\footnotetext{
${ }^{12}$ See, e.g., Feenstra (1989); Feenstra, Gagnon, and Knetter (1996); Yang (1997); Campa and Goldberg (2005); Hellerstein (2008); Gopinath, Itskhoki, and Rigobon (2010); Goldberg and Hellerstein (2013); Auer and Schoenle (2016); and Chen and Juvenal (2016) for empirical studies of exchange rate pass-through.
} 


\subsection{Elasticities and curvatures of demand and inverse de- mand}

Direct demand. Following Holmes (1989, p. 245), we define the own price elasticity of the firm's demand by $\epsilon_{F}(p) \equiv-(p / q(p)) \partial q_{j}(\mathbf{p}) /\left.\partial p_{j}\right|_{\mathbf{p}=(p, \ldots, p)}$ and the cross price elasticity by $\epsilon_{C}(p) \equiv(n-1)(p / q(p)) \partial q_{j^{\prime}}(\mathbf{p}) /\left.\partial p_{j}\right|_{\mathbf{p}=(p, \ldots, p)}$ for any distinct pair of indices $j$ and $j^{\prime}$. These are related to the industry demand elasticity $\epsilon(p)$ by $\epsilon_{F}(p)=\epsilon(p)+\epsilon_{C}(p){ }^{13}$

We define the curvature of the industry's direct demand $\alpha(p) \equiv-p q^{\prime \prime}(p) / q^{\prime}(p)$, as well as the own curvature $\alpha_{F}(p)$ of the firm's direct demand and the cross curvature $\alpha_{C}(p)$ of the firm's direct demand: ${ }^{14}$

$$
\begin{gathered}
\alpha_{F}(p) \equiv-\left.p\left(\frac{\partial q_{j}(\mathbf{p})}{\partial p_{j}}\right)^{-1} \frac{\partial^{2} q_{j}(\mathbf{p})}{\partial p_{j}^{2}}\right|_{\mathbf{p}=(p, \ldots, p)}, \\
\alpha_{C}(p) \equiv-\left.(n-1) p\left(\frac{\partial q_{j}(\mathbf{p})}{\partial p_{j}}\right)^{-1} \frac{\partial^{2} q_{j^{\prime}}(\mathbf{p})}{\partial p_{j} \partial p_{j^{\prime}}}\right|_{\mathbf{p}=(p, \ldots, p)},
\end{gathered}
$$

where $j$ and $j^{\prime}$ is an arbitrary pair of distinct indices. These curvatures satisfy $\alpha=\left(\alpha_{F}+\alpha_{C}\right) \epsilon_{F} / \epsilon$. They are related to the elasticity of $\epsilon_{F}(p)$ by $p \epsilon_{F}^{\prime}(p) / \epsilon_{F}(p)=$ $1+\epsilon(p)+\alpha_{F}(p)+\alpha_{C}(p) \cdot{ }^{15}$

Inverse demand. We define the own quantity elasticity of the firm's inverse demand $\eta_{F}(q) \equiv-(q / p(q)) \partial p_{j}(\mathbf{q}) /\left.\partial q_{j}\right|_{\mathbf{p}=(p, \ldots, p)}$ and the the cross quantity elasticity $\eta_{C}(q) \equiv(n-1)(q / p(q)) \partial p_{j^{\prime}}(\mathbf{q}) /\left.\partial q_{j}\right|_{\mathbf{p}=(p, \ldots, p)}$. These satisfy $\eta_{F}(q)=\eta(q)+\eta_{C}(q)$.

\footnotetext{
${ }^{13}$ Holmes (1989) shows this for two symmetric firms, but it is straightforward to verify this relation more generally. See the equation in Footnote 7.

${ }^{14}$ The curvature $\alpha_{F}(p)$ here corresponds to $\alpha(p)$ of Aguirre, Cowan, and Vickers (2010, p. 1603).

${ }^{15}$ This relationship can be verified as follows. The elasticity of the function $\epsilon_{F}(p)$ equals the sum of the elasticities of the three factors it is composed of:

$$
\frac{1}{\epsilon_{F}(p)} p \frac{d}{d p} \epsilon_{F}(p)=\frac{1}{p} p \frac{d}{d p} p+q(p) p \frac{d}{d p} \frac{1}{q(p)}+\left.\left(\frac{\partial q_{j}(\mathbf{p})}{\partial p_{j}}\right)^{-1}\right|_{\mathbf{p}=(p, \ldots, p)} p \frac{d}{d p}\left(\left.\frac{\partial q_{j}(\mathbf{p})}{\partial p_{j}}\right|_{\mathbf{p}=(p, \ldots, p)}\right) .
$$

The first elasticity on the right-hand side equals 1 , the second elasticity equals $\epsilon(p)$, and the third elasticity equals $\alpha_{F}(p)+\alpha_{C}(p)$, since

$$
\left.p \frac{d}{d p} \frac{\partial q_{j}(\mathbf{p})}{\partial p_{j}}\right|_{\mathbf{p}=(p, \ldots, p)}=\left.p \frac{\partial^{2} q_{j}(\mathbf{p})}{\partial p_{j}^{2}}\right|_{\mathbf{p}=(p, \ldots, p)}+\left.(n-1) p \frac{\partial^{2} q_{j}(\mathbf{p})}{\partial p_{j} \partial p_{j^{\prime}}}\right|_{\mathbf{p}=(p, \ldots, p)} .
$$
}


We define the curvature of the industry's inverse demand $\sigma(q) \equiv-q p^{\prime \prime}(q) / p^{\prime}(q)$, as well as the own curvature $\sigma_{F}(q)$ of the firm's inverse demand and the cross curvature $\sigma_{C}(q)$ of the firm's inverse demand by:

$$
\begin{gathered}
\sigma_{F}(q) \equiv-\left.q\left(\frac{\partial p_{j}(\mathbf{q})}{\partial q_{j}}\right)^{-1} \frac{\partial^{2} p_{j}(\mathbf{q})}{\partial q_{j}^{2}}\right|_{\mathbf{q}=(q, \ldots, q)}, \\
\sigma_{C}(q) \equiv-\left.(n-1) q\left(\frac{\partial p_{j}(\mathbf{q})}{\partial q_{j}}\right)^{-1} \frac{\partial^{2} p_{j^{\prime}}(\mathbf{q})}{\partial q_{j} \partial q_{j^{\prime}}}\right|_{\mathbf{q}=(q, \ldots, q)},
\end{gathered}
$$

for an arbitrary pair of distinct indices $j$ and $j^{\prime}$. These curvatures represent an oligopoly counterpart of monopoly $\sigma(q)$ in Aguirre, Cowan, and Vickers (2010, p. 1603). They satisfy the relationship $\sigma=\left(\sigma_{F}+\sigma_{C}\right) \eta_{F} / \eta$. They are related to the elasticity of $\eta_{F}(q)$ by $q \eta_{F}^{\prime}(q) / \eta_{F}(q)=1+\eta(q)+\sigma_{F}(q)+\sigma_{C}(q) .{ }^{16}$

\subsection{Expressions for pass-through and marginal cost of pub- lic funds}

In the case of price competition, the conduct index $\theta$ is $\theta=\epsilon / \epsilon_{F}=1 /\left(\eta \epsilon_{F}\right)$, which may be verified by comparing the firm's first-order condition with Equation 1.

\section{Proposition 7. Pass-through and marginal cost of public funds under} price competition. Under symmetric oligopoly with price competition and with a possibly non-constant marginal cost:

$$
\begin{aligned}
& \rho_{t}=\frac{1}{1-v} \frac{\epsilon_{F}}{\epsilon_{F}+\epsilon-\alpha_{F}-\alpha_{C}+\left(\frac{1-\tau}{1-v} \epsilon_{F}-1\right) \epsilon \chi}, \\
& \rho_{v}=\frac{1}{1-v} \frac{\epsilon_{F}-1}{\epsilon_{F}+\epsilon-\alpha_{F}-\alpha_{C}+\left(\frac{1-\tau}{1-v} \epsilon_{F}-1\right) \epsilon \chi} .
\end{aligned}
$$

For the marginal cost of public funds this implies:

$$
\begin{gathered}
M C_{t}=\frac{\left(1+\frac{1}{1-v} \epsilon_{F} \tau\right) \epsilon}{\epsilon-\alpha_{F}-\alpha_{C}+\frac{1}{1-v}(1-\epsilon \tau) \epsilon_{F}+\left(\frac{1-\tau}{1-v} \epsilon_{F}-1\right) \epsilon \chi}, \\
M C_{v}=\frac{\epsilon_{F}-1}{\epsilon_{F}} \frac{\left(1+\frac{1}{1-v} \epsilon_{F} \tau\right) \epsilon}{\epsilon_{F}+\epsilon-\alpha_{F}-\alpha_{C}+\frac{1}{1-v}\left(\epsilon_{F}-1\right)(v-\epsilon \tau)+\left(\frac{1-\tau}{1-v} \epsilon_{F}-1\right) \epsilon \chi} .
\end{gathered}
$$

\footnotetext{
${ }^{16}$ In analogy with Footnote 15, the elasticity of the function $\eta_{F}(q)$ is the sum of the elasticities of the three factors it is composed of, which are equal to $1, \eta(q)$, and $\sigma_{F}(q)+\sigma_{C}(q)$.
} 
In the case of constant marginal cost the pass-through expressions simplify to

$$
\rho_{t}=\frac{1}{1-v} \frac{\epsilon_{F}}{\epsilon_{F}+\epsilon-\alpha_{F}-\alpha_{C}}, \quad \rho_{v}=\frac{1}{1-v} \frac{\epsilon_{F}-1}{\epsilon_{F}+\epsilon-\alpha_{F}-\alpha_{C}} .
$$

Proof. Since in the case of price setting $\theta=\epsilon / \epsilon_{F}=1 /\left(\eta \epsilon_{F}\right)$, we have $(\eta+\chi) \theta=$ $(1+\epsilon \chi) / \epsilon_{F}$ and $(\theta \eta)^{\prime} \epsilon q=\epsilon q \frac{d}{d q}(\theta \eta)=\epsilon q \frac{d}{d q}\left(\epsilon_{F}^{-1}\right)=-\epsilon_{F}^{-2} \epsilon q \frac{d}{d q} \epsilon_{F}=\epsilon_{F}^{-2} p \frac{d}{d p} \epsilon_{F}=$ $\left(1+\epsilon-\alpha_{F}-\alpha_{C}\right) / \epsilon_{F}$, where in the last equality we utilized the expression for the elasticity of $\epsilon_{F}(p)$ from Subsection 3.1. Substituting these into the expression for $\rho_{t}$ in Proposition 6 gives

$$
\rho_{t}=\frac{1}{1-v} \frac{1}{1-\frac{1}{\epsilon_{F}}(1+\epsilon \chi)+\frac{1}{\epsilon_{F}}\left(1+\epsilon-\alpha_{F}-\alpha_{C}\right)+\frac{1-\tau}{1-v} \epsilon \chi},
$$

which is equivalent to the expression for $\rho_{t}$ in the proposition. Since for price setting $\theta=\epsilon / \epsilon_{F}$, the relationship in Proposition 4 implies $\rho_{v}=(\epsilon-\theta) \rho_{t} / \epsilon=\left(\epsilon_{F}-1\right) \rho_{t} / \epsilon_{F}$, which leads to the desired expression for $\rho_{v}$. The implication for the marginal cost of public funds follows by substituting these expressions into those of Propositions 1 and 2.

In the case of quantity competition, the conduct index $\theta$ is $\theta=\eta_{F} / \eta$, which may be verified by comparing the firm's first-order condition with 1 .

Proposition 8. Pass-through and marginal cost of public funds under quantity competition. Under symmetric oligopoly with quantity competition and with a possibly non-constant marginal cost:

$$
\begin{aligned}
& \rho_{t}=\frac{1}{1-v} \frac{\eta}{\eta+\left(1+\sigma_{C}+\sigma_{F}\right) \eta_{F}+\left(\frac{1-\tau}{1-v}-\eta_{F}\right) \chi}, \\
& \rho_{v}=\frac{1}{1-v} \frac{\left(1-\eta_{F}\right) \eta}{\eta+\left(1+\sigma_{C}+\sigma_{F}\right) \eta_{F}+\left(\frac{1-\tau}{1-v}-\eta_{F}\right) \chi} .
\end{aligned}
$$

For the marginal cost of public funds this implies:

$$
\begin{gathered}
M C_{t}=\frac{\eta_{F}+\frac{1}{1-v} \tau}{\eta+\left(1+\sigma_{C}+\sigma_{F}\right) \eta_{F}+\frac{1}{1-v}(v-\epsilon \tau) \eta+\left(\frac{1-\tau}{1-v}-\eta_{F}\right) \chi}, \\
M C_{v}=\frac{\left(1-\eta_{F}\right)\left(\eta_{F}+\frac{1}{1-v} \tau\right)}{\eta+\left(1+\sigma_{C}+\sigma_{F}\right) \eta_{F}+\left(\frac{1-\tau}{1-v}-\eta_{F}\right) \chi+\frac{1}{1-v}\left(1-\eta_{F}\right)(v-\epsilon \tau) \eta} .
\end{gathered}
$$


In the case of constant marginal cost the pass-through expressions simplify to

$$
\rho_{t}=\frac{1}{1-v} \frac{\eta}{\eta+\left(1+\sigma_{C}+\sigma_{F}\right) \eta_{F}}, \quad \rho_{v}=\frac{1}{1-v} \frac{\left(1-\eta_{F}\right) \eta}{\eta+\left(1+\sigma_{C}+\sigma_{F}\right) \eta_{F}} .
$$

Proof. In the case of quantity setting, $\theta=\eta_{F} / \eta$, so $(\eta+\chi) \theta=(1+\chi / \eta) \eta_{F}$ and $(\theta \eta)^{\prime} \epsilon q=q\left(\eta_{F}\right)^{\prime} / \eta=\left(1+\eta+\sigma_{C}+\sigma_{F}\right) \eta_{F} / \eta$, where in the last equality we utilized the expression for the elasticity of $\eta_{F}(q)$ from Subsection 3.1. Substituting these into the expression for $\rho_{t}$ in Proposition 6 gives

$$
\rho_{t}=\frac{1}{1-v} \frac{1}{1-\left(1+\frac{1}{\eta} \chi\right) \eta_{F}+\frac{1}{\eta}\left(1+\eta+\sigma_{C}+\sigma_{F}\right) \eta_{F}+\frac{1-\tau}{1-v} \frac{1}{\eta} \chi}
$$

After a rearrangement this gives the expression for $\rho_{t}$ in the proposition. Since $\theta=\eta_{F} / \eta$, Proposition 4 implies $\rho_{v}=(\epsilon-\theta) \rho_{t} / \epsilon=\left(1 / \eta-\eta_{F} / \eta\right) \rho_{t} \eta=\left(1-\eta_{F}\right) \rho_{t}$, which can be used to verify the expression for $\rho_{v}$. The implication for the marginal cost of public funds follows by substituting these expressions into those of Propositions 1 and 2 .

Cournot competition. Homogeneous-product Cournot competition is a very simple special case, where $\theta=1 / n, \eta=n \eta_{F}$, and $\sigma_{C}=(n-1) \sigma_{F}$.

Monopolistic competition. Monopolistic competition may be obtained by taking the large $n$ limit. As discussed in Weyl and Fabinger (2013, p. 544-546), in the case of quasi-linear utility of the form $U\left(\int u\left(q_{i}\right) d i\right)-p_{i} q_{i} d i$, it may be shown that $\theta=U^{\prime} u^{\prime \prime} /\left(U^{\prime \prime}\left(u^{\prime}\right)^{2}+U^{\prime} u^{\prime \prime}\right)$. With the most typical specification $u(q)=q^{\beta}$, $U(x)=x^{\gamma}$, this leads to a constant value of conduct index: $\theta=(1-\beta) /(1-\beta \gamma)$. Then $\eta=\eta_{F}(1-\beta \gamma) /(1-\beta)$.

\subsection{Simple Parametric Examples}

Tables 1 and 2 provide a summary of the variables of interest for linear demand and logit demand, respectively. ${ }^{17}$

\footnotetext{
${ }^{17} \mathrm{~A}$ discussion of the intuitive economic meaning of the formulas will be added to future versions of this paper.
} 


\begin{tabular}{|c|c|}
\hline Price setting & ty setting \\
\hline $\begin{array}{l}q_{j}\left(p_{1}, \ldots, p_{n}\right)=b-\lambda p_{j}+\mu \sum_{j^{\prime} \neq j} p_{j^{\prime}} \\
\epsilon_{F}=\frac{\lambda p}{q} \\
\epsilon_{C}=\frac{\mu(n-1) p}{q} \\
\theta=\frac{\lambda+\mu-\mu n}{\lambda} \\
p=\frac{b}{2 \lambda+\mu-\mu n}-\frac{\lambda(m c+t)}{(v-1)(2 \lambda+\mu-\mu n)} \\
q=\frac{b \lambda}{2 \lambda+\mu-\mu n}+\frac{\lambda(m c+t)(\lambda+\mu-\mu n)}{(v-1)(2 \lambda+\mu-\mu n)} \\
\rho_{t}=-\frac{\lambda-1)(2 \lambda+\mu-\mu n)}{(v-1)} \\
\rho_{v}=\frac{\lambda(m c+t)}{(v-1)(b(v-1)-\lambda(m c+t))} \\
M C_{t}=\quad \\
\frac{-(\lambda+\mu-\mu n)(\lambda p \tau+q(1-v))}{b \lambda \tau+q(-\lambda \tau+\mu(1-n)(v-1)+\lambda(v-2))} \\
M C_{v}= \\
\frac{-(\lambda+\mu-\mu n)(\lambda p-q)(\lambda p \tau+q(1-v))}{\lambda \tau(b-q)(\lambda p-q)+\lambda q(-\mu(n-1) p(v-1)+\lambda p(v-2)+q}\end{array}$ & $\begin{array}{l}q_{j}\left(p_{1}, \ldots, p_{n}\right)=b-\lambda p_{j}+\mu \sum_{j^{\prime} \neq j} p_{j^{\prime}} \\
\eta_{F}=-\frac{q(-\lambda-2 \mu+\mu n)}{p(\lambda+\mu)(\lambda+\mu-\mu n)} \\
\eta_{C}=\frac{q(\mu-\mu n)}{p(\lambda+\mu)(\lambda+\mu-\mu n)} \\
\theta=\frac{\lambda+2 \mu-\mu n}{\lambda+\mu} \\
p=\frac{b(\lambda-\mu(n-2))}{(2 \lambda-\mu(n-3))(\lambda+\mu-\mu n)}+\frac{(-\lambda-\mu)(m c+t)}{(v-1)(2 \lambda-\mu(n-3))} \\
q=\frac{(\lambda+\mu)(b(v-1)+(m c+t)(\lambda+\mu-\mu n))}{(v-1)(2 \lambda-\mu(n-3))} \\
\rho_{t}=\frac{-\lambda-\mu}{(v-1)(2 \lambda-\mu(n-3))} \\
\rho_{v}=\frac{\kappa(\lambda+\mu)(m c+t)}{(v-1)\left(b \kappa_{2}(v-1)-\kappa(\lambda+\mu)(m c+t)\right)} \\
M C_{t}= \\
\frac{q(v-1)(\lambda-\mu(n-2))-p \tau(\lambda+\mu)(\lambda+\mu-\mu n)}{p \tau(\lambda+\mu)(\lambda+\mu-\mu n)+\mu q(n(-v)+n+2 v-3)+\lambda q(v-2)} \\
M C_{v}=\frac{1}{\lambda+\mu} \times \\
\frac{(\mu(n-2) q+\kappa p(\lambda+\mu)+\lambda(-q))\left(\kappa_{2} q(v-1)-\kappa p \tau(\lambda+\mu)\right)}{\kappa p(q(\mu(n-3)+\mu(n-2)(\tau-v)+\lambda(v-\tau-2))+\kappa p \tau(\lambda+\mu))+\kappa_{2} q^{2} v}\end{array}$ \\
\hline
\end{tabular}

Table 1: Linear demand summary. Here $\kappa \equiv \lambda-(n-1) \mu$ and $\kappa_{2} \equiv \lambda-(n-2) \mu$.

\begin{tabular}{l|l}
\hline \multicolumn{1}{c|}{ Price setting } & \multicolumn{1}{c}{ Quantity setting } \\
\hline$q_{j}\left(p_{1}, \ldots, p_{n}\right)=\frac{\exp \left(\delta-\beta p_{j}\right)}{1+\sum_{j^{\prime}=1, \ldots, n} \exp \left(\delta-\beta p_{j^{\prime}}\right)}$ & $q_{j}\left(p_{1}, \ldots, p_{n}\right)=\frac{\exp \left(\delta-\beta p_{j}\right)}{1+\sum_{j^{\prime}=1, \ldots, n} \exp \left(\delta-\beta p_{j^{\prime}}\right)}$ \\
$p_{j}\left(s_{1}, \ldots, s_{n}\right)=\frac{1}{\beta}\left(\delta-\log \frac{s_{j}}{s_{0}}\right)$ & $p_{j}\left(s_{1}, \ldots, s_{n}\right)=\frac{1}{\beta}\left(\delta-\log \frac{s_{j}}{s_{0}}\right)$ \\
$\epsilon_{F}=\beta(1-s) p$ & $\eta_{F}=\frac{1-(n-1) s}{1-n s}\left(\delta-\log \frac{s}{1-n s}\right)^{-1}$ \\
$\epsilon_{C}=\beta(n-1) p s$ & $\eta_{C}=-\frac{(n-1) s}{1-n s}\left(\delta-\log \frac{s}{1-n s}\right)^{-1}$ \\
$\theta=\frac{1-n s}{1-s}$ & $\theta=1-(n-1) s$ \\
$p=\frac{m c+t}{1-v}+\frac{1}{\beta(1-s(p))}$ & $\frac{m c+t}{1-v}=\frac{1-\delta+s((\delta-1) n+1)+(1-n s) \log \frac{s}{1-n s}}{\beta(n s-1)}$ \\
$\rho_{t}=\frac{(s-1)^{2}}{(1-v)\left(1-s-(n-1) s^{2}\right)}$ & $\rho_{t}=\frac{1}{(1-v)\left(1+s(1-n s)^{-1}\right)}$ \\
\hline
\end{tabular}

Table 2: Logit demand summary. We define $s_{0}=1-\sum_{j=1, \ldots, n} s_{j}<1$. Additional expressions will be added to this table in future versions of this draft. 


\section{Oligopoly with Multi-Product Firms}

In this section, we argue that the results above can be extended to the case of multiproduct firms just by a reinterpretation of the same formulas (without modifying them). Assume there are $n_{p}$ product categories, and the demand for firm $j$ 's $k$-th product is given by $q_{j k}=q_{j k}\left(\mathbf{p}_{1}, \mathbf{p}_{2}, . ., \mathbf{p}_{n}\right)$, where $\mathbf{p}_{j}=\left(p_{j 1}, \ldots, p_{j k}, \ldots, p_{j K}\right)$ for each $j=1,2, \ldots, n{ }^{18}$ The firms are symmetric, and for each firm, the product it produces are also symmetric. The firm's profit per product is

$$
\pi_{j}=\frac{1}{n_{p}} \sum_{k=1}^{n_{p}}\left((1-v) p_{j k} q_{j k}-t q_{j k}-c\left(q_{j k}\right)\right) .
$$

We work with an equilibrium in which any firm $j$ sets a uniform price $p_{j}$ for all of its products: $p_{j k}=p_{j}$, and consequently sells an amount $q_{j}$ of each of them: $q_{j k}=q_{j}{ }^{19}$ In this case the profit per product equals $\pi_{j}=(1-v) p_{j} q_{j}-t q_{j}-c\left(q_{j}\right)$, which is formally the same as for single-product firms. For this reason, we can identify the prices $p_{j}$ and quantities $q_{j}$ of Section 2 with the prices $p_{j}$ and quantities $q_{j}$ introduced here in this paragraph. The discussion in Section 2 was general and applies to this case of symmetric oligopoly with multi-product firms as well. We can use the same definitions for the variables of interest, including the industry demand elasticity $\epsilon$ and the conduct index $\theta$.

The definitions and results for the cases of price competition and quantity competition discussed Section 3 are also applicable here. It may be useful to translate some of the most important variables of that discussion into product-level variables.

\footnotetext{
${ }^{18}$ See, e.g., Nocke and Schutz (2016) for a recent treatment of multi-product oligopoly.

${ }^{19}$ For brevity, we do not explicitly discuss the standard conditions for the existence and uniqueness of non-cooperative Nash equilibria of the different underlying oligopoly games.
} 
For derivatives of the direct demand system, we introduce the notation ${ }^{20}$

$$
\begin{array}{cccc}
\xi_{1} \equiv \frac{\partial q_{j k}}{\partial p_{j k}}, & \xi_{0,1} \equiv \frac{\partial q_{j k}}{\partial p_{j k^{\prime}}}, & \\
\xi_{2} \equiv \frac{\partial q_{j k}}{\partial p_{j k}^{2}}, & \xi_{1,1} \equiv \frac{\partial q_{j k}}{\partial p_{j k} \partial p_{j k^{\prime}}}, & \xi_{0,2} \equiv \frac{\partial q_{j k}}{\partial p_{j k^{\prime}}^{2}}, & \xi_{0,1,1} \equiv \frac{\partial q_{j k}}{\partial p_{j k^{\prime}} \partial p_{j k^{\prime \prime}}}, \\
\tilde{\xi}_{2} \equiv \frac{\partial q_{j k}}{\partial p_{j k} \partial p_{j^{\prime} k}} & \tilde{\xi}_{1,1} \equiv \frac{\partial q_{j k}}{\partial p_{j k} \partial p_{j^{\prime} k^{\prime}}}, & \tilde{\xi}_{0,2} \equiv \frac{\partial q_{j k}}{\partial p_{j k^{\prime}} \partial p_{j^{\prime} k^{\prime}}}, & \tilde{\xi}_{0,1,1} \equiv \frac{\partial q_{j k}}{\partial p_{j k^{\prime}} \partial p_{j^{\prime} k^{\prime \prime}}},
\end{array}
$$

where the derivatives are evaluated at the fully symmetric point, where any $p_{j k}$ equals the common value $p$. For specific choices of the demand system, these derivatives can be closely related. For example, if the substitution pattern between two goods produced by two different firms does not depend on the identity of the goods, then $\tilde{\xi}_{2}=\tilde{\xi}_{0,2}=\tilde{\xi}_{1,1}=\tilde{\xi}_{0,1,1}$. In terms of these derivatives, we can write

$$
\begin{gathered}
\epsilon_{F}=-\frac{p}{q}\left(\xi_{1}+\left(n_{p}-1\right) \xi_{0,1}\right), \\
\epsilon=-\frac{p}{q}\left(\xi_{1}+\left(n_{p}-1\right) \xi_{0,1}+(n-1) \tilde{\xi}_{1}+(n-1)\left(n_{p}-1\right) \tilde{\xi}_{0,1}\right), \\
\alpha_{F}=\frac{p^{2}}{q \epsilon_{F}}\left(\xi_{2}+\left(n_{p}-1\right)\left(\xi_{1,1}+\xi_{0,2}+\left(n_{p}-2\right) \xi_{0,1,1}\right)\right), \\
\alpha_{C}=(n-1) \frac{p^{2}}{q \epsilon_{F}}\left(\tilde{\xi}_{2}+\left(n_{p}-1\right)\left(\tilde{\xi}_{1,1}+\tilde{\xi}_{0,2}+\left(n_{p}-2\right) \tilde{\xi}_{0,1,1}\right)\right) .
\end{gathered}
$$

These can be substituted into the results of Proposition 7 to find the pass-through and the marginal cost of public funds under price competition.

For the inverse demand system the analogous definitions are

$$
\begin{aligned}
& \zeta_{1} \equiv \frac{\partial q_{j k}}{\partial p_{j k}}, \quad \zeta_{0,1} \equiv \frac{\partial q_{j k}}{\partial p_{j k^{\prime}}}, \\
& \zeta_{2} \equiv \frac{\partial q_{j k}}{\partial p_{j k}^{2}}, \quad \zeta_{1,1} \equiv \frac{\partial q_{j k}}{\partial p_{j k} \partial p_{j k^{\prime}}}, \quad \zeta_{0,2} \equiv \frac{\partial q_{j k}}{\partial p_{j k^{\prime}}^{2}}, \quad \zeta_{0,1,1} \equiv \frac{\partial q_{j k}}{\partial p_{j k^{\prime}} \partial p_{j k^{\prime \prime}}}, \\
& \tilde{\zeta}_{2} \equiv \frac{\partial q_{j k}}{\partial p_{j k} \partial p_{j^{\prime} k}} \quad \tilde{\zeta}_{1,1} \equiv \frac{\partial q_{j k}}{\partial p_{j k} \partial p_{j^{\prime} k^{\prime}}}, \quad \tilde{\zeta}_{0,2} \equiv \frac{\partial q_{j k}}{\partial p_{j k^{\prime}} \partial p_{j^{\prime} k^{\prime}}}, \quad \tilde{\zeta}_{0,1,1} \equiv \frac{\partial q_{j k}}{\partial p_{j k^{\prime}} \partial p_{j^{\prime} k^{\prime \prime}}} \text {. }
\end{aligned}
$$

The relations

$$
\begin{gathered}
\eta_{F}=-\frac{q}{p}\left(\zeta_{1}+\left(n_{p}-1\right) \zeta_{0,1}\right) \\
\eta=-\frac{q}{p}\left(\zeta_{1}+\left(n_{p}-1\right) \zeta_{0,1}+(n-1) \tilde{\zeta}_{1}+(n-1)\left(n_{p}-1\right) \tilde{\zeta}_{0,1}\right) \\
\sigma_{F}=\frac{q^{2}}{p \eta_{F}}\left(\zeta_{2}+\left(n_{p}-1\right)\left(\zeta_{1,1}+\zeta_{0,2}+\left(n_{p}-2\right) \zeta_{0,1,1}\right)\right), \\
\sigma_{C}=(n-1) \frac{q^{2}}{p \eta_{F}}\left(\tilde{\zeta}_{2}+\left(n_{p}-1\right)\left(\tilde{\zeta}_{1,1}+\tilde{\zeta}_{0,2}+\left(n_{p}-2\right) \tilde{\zeta}_{0,1,1}\right)\right) .
\end{gathered}
$$

can be substituted into the results of Proposition 8 to find the pass-through and marginal cost of public funds under price competition.

\footnotetext{
${ }^{20}$ In this notation, the first subscript counts the derivatives with respect to the relevant price with index $k$, the second subscript counts the derivatives with respect to the price with index $k^{\prime}$ distinct from $k$, and the third subscript counts derivatives respect to the price with index $k^{\prime \prime}$ distinct from both $k$ and $k^{\prime}$. Further, $\xi$ corresponds to derivatives with respect to prices charged by the same firm $j$, while $\tilde{\xi}$ corresponds to derivatives with respect to prices charged by firm $j$ and some other firm $j^{\prime}$.
} 


\section{Multi-Dimensional Pass-Through Framework}

In this section we generalize our previous results to a significantly more general specification of taxation that involves multiple tax parameters. We define two different types of pass-through vectors: the pass-through rate vector and the pass-through quasi-elasticity vector. We study their properties and show that they play a central role in evaluating welfare changes in response to changes in taxation.

\subsection{Pass-through, conduct index, and welfare: a general dis- cussion}

\subsubsection{Generalized pass-through and tax sensitivities}

Consider a tax structure under which a firm's tax payment may be expressed as $\phi(p, q, \mathbf{T})$, where $\mathbf{T} \equiv\left(T_{1}, \ldots, T_{d}\right)$ is a $d$-dimensional vector of tax parameters. ${ }^{21}$ The components of the (per-firm) tax revenue gradient vector

$$
\mathbf{f} \equiv \nabla \phi(p, q, \mathbf{T})
$$

are

$$
\phi_{T_{\ell}}(p, q, \mathbf{T}) \equiv \frac{\partial \phi(p, q, \mathbf{T})}{\partial T_{\ell}}
$$

Here, as in other parts of the paper, we use the symbol $\nabla$ for the $d$-dimensional gradient with respect to $\mathbf{T}$. The arguments $p$ and $q$ in $\phi(p, q, \mathbf{T})$ are treated as fixed for the purposes of taking this gradient. We denote the equilibrium price function ${ }^{22}$ by $p^{\star}(\mathbf{T})$ and its gradient, the pass-through rate vector, by

$$
\tilde{\boldsymbol{\rho}} \equiv \nabla p^{\star}(\mathbf{T})
$$

Further, we use the components of the $\mathbf{f}$ and $\tilde{\boldsymbol{\rho}}$ to define the pass-through quasielasticity vector as

$$
\boldsymbol{\rho} \equiv\left(\rho_{T_{1}}, \ldots, \rho_{T_{d}}\right), \quad \rho_{T_{\ell}} \equiv q \frac{\tilde{\rho}_{T_{\ell}}}{f_{T_{\ell}}}=\frac{q}{\phi_{T_{\ell}}(p, q, \mathbf{T})} \frac{\partial p^{\star}}{\partial T_{\ell}} .
$$

\footnotetext{
${ }^{21}$ To be precise, $\phi(p, q, \mathbf{T})$ represents a simplified notation for a function $\phi\left(p, q, T_{1}, \ldots, T_{d}\right)$ with $d+2$ arguments.

${ }^{22}$ Unlike the inverse demand function $p(q)$, the function $p^{\star}(\mathbf{T})$ takes the vector of taxes as arguments and its functional value is the price in the resulting equilibrium.
} 
Note that the components of $\boldsymbol{\rho}$ are all dimensionless. We define the (first-order) price sensitivity $\nu$ of the tax revenue and the (first-order) quantity sensitivity $\tau$ of the (per-firm) tax revenue as follows:

$$
\nu(p, q, \mathbf{T}) \equiv \frac{1}{q} \phi_{p}(p, q, \mathbf{T}), \quad \tau(p, q, \mathbf{T}) \equiv \frac{1}{p} \phi_{q}(p, q, \mathbf{T})
$$

Their derivatives are

$$
\nu_{T_{\ell}}(p, q, \mathbf{T}) \equiv \frac{\partial \nu(p, q, \mathbf{T})}{\partial T_{\ell}}, \quad \tau_{T_{\ell}}(p, q, \mathbf{T}) \equiv \frac{\partial \tau(p, q, \mathbf{T})}{\partial T_{\ell}} .
$$

The analogous definitions for second-order sensitivities are:

$\nu_{(2)}(p, q, \mathbf{T}) \equiv \frac{p}{q} \frac{\partial^{2} \phi(p, q, \mathbf{T})}{\partial p^{2}}, \tau_{(2)}(p, q, \mathbf{T}) \equiv \frac{q}{p} \frac{\partial^{2} \phi(p, q, \mathbf{T})}{\partial q^{2}}, \kappa(p, q, \mathbf{T}) \equiv \frac{\partial^{2} \phi(p, q, \mathbf{T})}{\partial p \partial q}$.

The first-order and second-order sensitivities are dimensionless, just like the components of $\boldsymbol{\rho}$. In this section we keep the same definition of the elasticities $\epsilon$ and $\eta$ as before.

\subsubsection{Generalized conduct index}

We introduce the conduct index $\theta$ as a function independent of the cost-side of the oligopoly game such that in equilibrium the following condition holds:

$$
(1-\tau-(1-\nu) \eta \theta) p=m c .
$$

In the case of unit and ad-valorem taxation, this definition reduces to the conduct index defined earlier. In principle, there are many possible definitions that agree with the earlier definition in the case of unit and ad-valorem taxation, but we found the specification of Equation 3 particularly convenient.

\subsubsection{Relative size of the components of pass-through vectors}

Proposition 9. The pass-through rates and quasi-elasticities satisfy ${ }^{23}$

$$
\frac{\tilde{\rho}_{T_{\ell}}}{\tilde{\rho}_{T_{\ell^{\prime}}}}=\frac{\tau_{T_{\ell^{\prime}}}-\nu_{T_{\ell^{\prime}}} \eta \theta}{\tau_{T_{\ell}}-\nu_{T_{\ell}} \eta \theta}, \quad \frac{\rho_{T_{\ell}}}{\rho_{T_{\ell^{\prime}}}}=\frac{f_{T_{\ell^{\prime}}}}{f_{T_{\ell}}} \frac{\tau_{T_{\ell}}-\eta \theta \nu_{T_{\ell}}}{\tau_{T_{\ell^{\prime}}}-\eta \theta \nu_{T_{\ell^{\prime}}}} .
$$

\footnotetext{
${ }^{23}$ If the denominators are zero, the fractions become ill-defined. In that case, of course, the statement does not apply.
} 
Proof. Consider an infinitesimal tax change such that the equilibrium price (and therefore quantity) does not change: $\tilde{\boldsymbol{\rho}} \cdot d \mathbf{T}=0$. Let us choose $d \mathbf{T}$ to have just two non-zero components: $d T_{\ell}$ and $d T_{\ell^{\prime}}$. This implies

$$
\frac{\tilde{\rho}_{T_{\ell}}}{\tilde{\rho}_{T_{\ell^{\prime}}}}=-\frac{d T_{\ell^{\prime}}}{d T_{\ell}}
$$

Since Equation 3 must hold both before and after the tax change, we must be that $1-\tau-(1-\nu) \eta \theta$ does not change, and in turn

$$
\left(-\tau_{T_{\ell}}+\nu_{T_{\ell}} \eta \theta\right) d T_{\ell}+\left(-\tau_{T_{\ell^{\prime}}}+\nu_{T_{\ell^{\prime}}} \eta \theta\right) d T_{\ell^{\prime}}=0
$$

Substituting for $d T_{\ell^{\prime}}$ from this equation into Equation 4 and using the definition of pass-through quasi-elasticities leads to the desired result.

Since the components have known proportions, we can write them using a common factor $p \rho_{(0)}$ as

$$
\begin{gathered}
\tilde{\rho}_{T_{\ell}}=\left(\tau_{T_{\ell}}-\nu_{T_{\ell}} \eta \theta\right) p \rho_{(0),}, \\
\rho_{T_{\ell}}=\frac{p q}{f_{T_{\ell}}}\left(\tau_{T_{\ell}}-\nu_{T_{\ell}} \eta \theta\right) \rho_{(0)} .
\end{gathered}
$$

\subsubsection{Absolute size of the components of pass-through vectors}

Proposition 10. The value of the factor $\rho_{(0)}$ introduced above is given by the formula:

$$
\frac{1}{\rho_{(0)}}=1-\kappa+\epsilon \tau_{(2)}+(1-\tau) \epsilon \chi+\left(\nu-\kappa+\eta \nu_{(2)}+(\omega-\eta-\chi)(1-\nu)\right) \theta,
$$

where $\omega \equiv q(\eta \theta)^{\prime} /(\eta \theta)$, with the prime denoting a derivative with respect to the quantity $q$.

Proof. The same type of reasoning as in the proof of Proposition 6 is useful here. In particular, comparative statics of Equation 3 with respect to a tax $T_{\ell}$ leads to the desired result after utilizing the definitions above and eliminating marginal cost using, again, Equation 3. The calculation is a bit tedious, but completely straightforward. 


\subsubsection{Welfare changes and their relationship to pass-through vectors}

Welfare component changes in response to an infinitesimal change in taxes may be found as follows. The consumer surplus change in response to an infinitesimal change $d T_{\ell}$ in the tax $T_{\ell}$ is

$$
d C S=-q d p=-q \tilde{\rho}_{T_{\ell}} d T_{\ell},
$$

which means that in vector notation, $\frac{1}{q} \nabla C S=-\tilde{\boldsymbol{\rho}}$. The change in producer surplus (firm profit) is

$$
d P S=d(p q-c(q)-\phi(p, q, \mathbf{T}))=\left(\phi_{T_{\ell}}(p, q, \mathbf{T})-(1-\nu)(1-\theta) \tilde{\rho}_{T_{\ell}}\right) d T_{\ell},
$$

where we utilized Equation 3 to eliminate marginal cost. In vector notation, this is $\frac{1}{q} \nabla P S=(1-\nu)(1-\theta) \tilde{\boldsymbol{\rho}}-\mathbf{f}$, since $\mathbf{f}=\nabla_{\mathbf{T}} \phi(p, q, \mathbf{T})$. The change in tax revenue is

$d R=\phi_{p}(p, q, \mathbf{T}) d p+\phi_{q}(p, q, \mathbf{T}) d q+\phi_{T_{\ell}}(p, q, \mathbf{T}) d T_{\ell}=\left(\phi_{T_{\ell}}(p, q, \mathbf{T})-(\epsilon \tau-\nu) \tilde{\rho}_{T_{\ell}}\right) d T_{\ell}$.

In vector notation, $\frac{1}{q} \nabla R=\mathbf{f}-(\epsilon \tau-\nu) \tilde{\boldsymbol{\rho}}$. Finally, for the change in social welfare, we have

$$
d W=(p-m c) d q=(\epsilon \tau+\theta(1-\nu)) \tilde{\rho}_{T_{\ell}} d T_{\ell}
$$

In vector notation, $\frac{1}{q} \nabla W=-(\epsilon \tau+\theta(1-\nu)) \tilde{\boldsymbol{\rho}}$.

Note that the welfare components $C S(\mathbf{T}), P S(\mathbf{T}), R(\mathbf{T})$, and $W(\mathbf{T})=$ $C S(\mathbf{T})+P S(\mathbf{T})+R(\mathbf{T})$ are all treated as functions of taxes only and represent the equilibrium outcomes. This is different from the tax revenue function $\phi(p, q, \mathbf{T})$, which has also $p$ and $q$ as arguments and which is specified by the government irrespective of the equilibrium. We summarize these findings in the following proposition.

Proposition 11. The tax gradients of consumer surplus, producer surplus, tax revenue, and social welfare with respect to the taxes all belong to a two-dimensional vector space spanned by $\mathbf{f}$ and $\tilde{\boldsymbol{\rho}}$. The precise linear combinations of $\mathbf{f}$ and $\tilde{\boldsymbol{\rho}}$ are

$$
\frac{1}{q} \nabla C S=-\tilde{\boldsymbol{\rho}},
$$




$$
\begin{gathered}
\frac{1}{q} \nabla P S=(1-\nu)(1-\theta) \tilde{\boldsymbol{\rho}}-\mathbf{f}, \\
\frac{1}{q} \nabla R=\mathbf{f}+(\nu-\epsilon \tau) \tilde{\boldsymbol{\rho}}, \\
\frac{1}{q} \nabla W=-((1-\nu) \theta+\epsilon \tau) \tilde{\boldsymbol{\rho}} .
\end{gathered}
$$

These relationships, considered component-wise, immediately imply the following results for welfare change ratios. ${ }^{24}$

Proposition 12. The marginal cost of public funds of a tax $T_{\ell}, M C_{T_{\ell}}=(\nabla R)_{T_{\ell}} /(\nabla W)_{T_{\ell}}$, is

$$
M C_{T_{\ell}}=\frac{(1-\nu) \theta+\epsilon \tau}{\frac{1}{\rho_{T_{\ell}}}+\nu-\epsilon \tau} .
$$

The incidence of this tax, $I_{T_{\ell}}=(\nabla C S)_{T_{\ell}} /(\nabla P S)_{T_{\ell}}$, equals:

$$
I_{T_{\ell}}=\frac{1}{\frac{1}{\rho_{T_{\ell}}}-(1-\nu)(1-\theta)} .
$$

Similarly, the social incidence, $S I_{T_{\ell}}=(\nabla W)_{T_{\ell}} /(\nabla P S)_{T_{\ell}}$, equals:

$$
S I_{T_{\ell}}=\frac{(1-\nu) \theta+\epsilon \tau}{\frac{1}{\rho_{T_{\ell}}}-(1-\nu)(1-\theta)} .
$$

\subsection{Pass-through, conduct index, and welfare: special cases}

The results of the previous subsection contain our results for ad valorem and unit taxes as special cases, but offer much greater generality, since the taxes (government interventions) may be specified in a very flexible way. In fact, it may be shown the symmetric oligopoly results in Weyl and Fabinger (2013) are also special cases of the present results. In particular, the analysis there considers either unit taxes or exogenous competition (an exogenous quantity supplied to the market). The case of unit taxes are clearly included in the present results (and motivated this paper). But it turns out that the case of exogenous competition is included as well. The reasoning is as follows.

\footnotetext{
${ }^{24}$ Remember that the $T_{\ell}$ component of the vector $\mathbf{f}$ is $\phi_{T_{\ell}}(p, q, \mathbf{T})=q \tilde{\rho}_{T_{\ell}} / \rho_{T_{\ell}}$.
} 
Consider a tax $T_{1}=\tilde{q}$ of the form

$$
\phi(p, q, \tilde{q})=\tilde{q} p+c(q-\tilde{q})-c(q)
$$

The firm's profit is then

$$
p q-c(q)-\phi(p, q, \tilde{q})=p(q-\tilde{q})+c(q-\tilde{q}) .
$$

The firm, therefore, has the same profit function as in the case of exogenous competition $\tilde{q}$ in Weyl and Fabinger (2013). Proposition 12 (specialized to constant marginal cost and zero initial $\tilde{q}$ ) then implies the social incidence result in Principle of Incidence 3 in Weyl and Fabinger (2013, p. 548).

Similarly, the relationships between pass-through of unit taxes and of exogenous competition are implied by the general result of Proposition 9 for the tax specification $T_{1}=t, T_{2}=\tilde{q}$,

$$
\phi(p, q, t, \tilde{q})=t q+\tilde{q} p+c(q-\tilde{q})-c(q) .
$$

To obtain the absolute size of the two types of pass-through, one can straightforwardly use Proposition 10.

\section{Concluding Remarks}

In this paper, we characterize the welfare burden of taxation and the tax incidence in symmetric oligopoly with a general specification of competition, demand and cost. We first derive formulas for marginal welfare losses from unit and ad valorem taxation, $M C_{t}$ and $M C_{v}$, using the unit tax pass-through rate $\rho_{t}$ and the ad valorem tax pass-through semi-elasticity $\rho_{v}$. We show that $\rho_{v}$ can be expressed in terms of $\rho_{t}$. These relationships are used to derive sufficient statistics for $M C_{t}$ and $M C_{v}$. In the case of price or quantity competition, we explain how $\rho_{t}$ and $\rho_{v}$ can be written only in terms of the demand elasticities, the demand curvatures, and the marginal cost elasticity. We discuss relationships to other quantities of interest, as well as illustrative special cases. We also show that these results have a very natural 
generalizations to a general specification of the tax revenue function as a function parametrized by a vector of tax parameters.

In follow-up work (or in future versions of this paper), we plan to discuss how this framework generalizes to other markets. We will provide an extension of our analysis to the case of asymmetric oligopoly, where the firms face different costs and possibly also different taxes. ${ }^{25,26}$ This discussion is analogous, but since it would require a lengthy explanation, we did not include it in this version of the paper. Similarly, we plan to extend the analysis to the case of supply chains (see Peitz and Reisinger (2014)). Other possible directions include the case of two-sided platform competition (White and Weyl (2016)) or the case of the interactive effects of taxation for multiple imperfectly competitive product markets. ${ }^{27,28}$ In addition, it would be of interest to develop flexible, but analytically solvable examples along the lines of Fabinger and Weyl (2016).

\footnotetext{
${ }^{25}$ By allowing (constant) asymmetric marginal costs, Anderson, de Palma, and Kreider (2001b) show that under quantity competition with homogeneous products (i.e., Cournot competition), ad valorem taxation is still preferable to unit taxation, although they were not able to verify if the same conclusion held under quantity competition with product differentiation. However, Anderson, de Palma, and Kreider (2001b) discuss a specific demand system (with perfectly inelastic individual demand) under which unit taxation is preferable to ad valorem taxation if the required tax revenue is sufficiently high. We conjecture that one could obtain further generalization by allowing the conduct index $\theta$ to be firm-specific. See also Zimmerman and Carlson (2010) for a parametric analysis of asymmetric firms.

${ }^{26}$ Interestingly, Tremblay and Tremblay (2016) study tax incidence in a duopoly where one firm competes in price and the other firm competes in quantity, focusing on unit taxation. The passthrough rates can be different for the two identical firms (in terms of demand and cost): the quantity-competing firm has a higher pass-through rate than the price-competing firm has. This is in contrast with the result that the pass-through rate under price competition is generally higher.

${ }^{27}$ Among many others, Ballard, Shoven, and Whalley (1985) study this issue for perfectly competitive markets.

${ }^{28}$ In addition, our methodology could be utilized to study other important issues of pricing in general such as welfare effects of oligopolistic third-degree price discrimination (Aguirre, Cowan, and Vickers (2010) is a study of monopoly). One may also study, for example, advertising pass-through (Draganska and Vitorino (2015)): the firm's demand can be modeled as $q_{j}=q_{j}\left(p_{1}, \ldots, p_{n} ; a_{1}, \ldots, a_{n}\right)$, where $a_{j}$ is firm $j$ 's investment in advertising. Free-riding, because of the spillover effect, may be more or less serious depending on demand elasticities and curvatures.
} 


\section{References}

Adachi, T., Ebina, T., 2014a. Double marginalization and cost pass-through: WeylFabinger and Cowan meet Spengler and Bresnahan-Reiss. Economics Letters, 122(2), $170-175$.

Adachi, T., Ebina, T., 2014b. Cost pass-through and inverse demand curvature in vertical relationships with upstream and downstream competition. Economics Letters, 124(3), 465-468.

Aguirre, I., Cowan, S., Vickers, J., 2010. Monopoly price discrimination and demand curvature. American Economic Review, 100(4), 1601-1615.

Alexandrov, A. and Bedre-Defolie, Ö., 2017. LeChatelier-Samuelson principle in games and pass-through of shocks. Journal of Economic Theory, March 2017

Anderson, S. P., de Palma, A., Kreider, B., 2001a. Tax incidence in differentiated product oligopoly. Journal of Public Economics, 81(2), 173-192.

Anderson, S. P., de Palma, A., Kreider, B., 2001b. The efficiency of indirect taxes under imperfect competition. Journal of Public Economics, 81(2), 231-251.

Anderson, S. P., de Palma, A., Thisse, J.-F., 1992. Discrete choice theory of product differentiation. The MIT Press.

Atkin, D., Donaldson, D., 2016. Who's getting globalized? The size and implications of intra-national trade costs. Manuscript.

Auer, R. A., Schoenle, R.S., 2016. Market structure and exchange rate pass-through. Journal of International Economics, 98, 60-77.

Ballard, C. L., Shoven, J.B., Whalley, J., 1985. General equilibrium computations of the marginal welfare costs of taxes in the United States. American Economic Review, 75(1), 128-138.

Berry, S. T., 1994. Estimating discrete-choice models of product differentiation. RAND Journal of Economics, 25(2), 242-262. 
Bonnet, C., Dubois, P., Villas-Boas, S.B., Klapper, D., 2013. Empirical evidence on the role of nonlinear wholesale pricing and vertical restraints on cost pass-through. Review of Economics and Statistics, 95(2), 500-515.

Bonnet, C., Réquillart, V., 2013. Tax incidence with strategic firms in the soft drink market. Journal of Public Economics, 106, 77-88.

Bresnahan, T. F., 1989. Empirical studies of industries with market power. In R. Schmalensee and R.D. Willig (eds.), Handbook of industrial organization, Vol. 2. Amsterdam, The Netherlands: Elsevier Science Publishers B.V., 1011-1057.

Browning, E. K., 1976. The marginal cost of public funds. Journal of Political Economy, 84(2), 283-298.

Buchanan, J. M., Tullock, G., 1965. Public and private interaction under reciprocal externality. In J. Margolis (ed.), The public economy of urban communities, Resources for the Future, 52-73.

Campa, J. M., Goldberg, L.S., 2005. Exchange rate pass-through into import prices. Review of Economics and Statistics, 87(4), 679-690.

Campos-Vázquez, R. M., Medina-Cortina, E.M., 2015. Pass-through and competition: The impact of soft drink taxes using Mexican supermarket stores. Manuscript.

Chen, N., Juvenal, L., 2016. Quality, trade, and exchange rate pass-through. Journal of International Economics, 100, 61-80.

Chen, Y., Schwartz, M., 2015. Differential pricing when costs differ: A welfare analysis. RAND Journal of Economics, 46(2), 442-460.

Chetty, R., 2009. Sufficient Statistics for Welfare Analysis: A Bridge between Structural and Reduced-Form Methods. Ann. Rev. Econ. 1:451-88.

Corts, K. S., 1999. Conduct parameters and the measurement of market power. Journal of Econometrics, 88(2), 227-250. 
Cowan, S., 2012. Third-degree price discrimination and consumer surplus. Journal of Industrial Economics, 6(2), 333-345.

Cowan, S., 2016. Welfare-increasing third-degree price discrimination. RAND Journal of Economics, 47(2), 326-340.

Delipalla, S., Keen, M., 1992. The comparison between ad valorem and specific taxation under imperfect competition. Journal of Public Economics, 49(3), 351-367.

Delipalla, S., O’Donnell, O., 2001. Estimating tax incidence, market power, and market conduct: The European cigarette industry. International Journal of Industrial Organization, 19(6), 885-908.

Draganska, M., Vitorino, M. A., 2015. Advertising pass-through: How does advertising affect retail prices? Manuscript.

Duso, T., Szücs, F., 2016. Market power and heterogeneous pass-through in German electricity retail. Manuscript.

Fabinger, M., Weyl, E. G., 2016. The average-marginal relationship and tractable equilibrium forms. Manuscript.

Fabra, N., Reguant, M., 2014. Pass-through of emissions costs in electricity markets. American Economic Review, 104(9), 2872-2899.

Feenstra, R. C., 1989. Symmetric pass-through of tariffs and exchange rates under imperfect competition: An empirical test. Journal of International Economics, $27(1-2), 25-45$.

Feenstra, R. C., Gagnon, J. E., Knetter, M. M., 1996. Market share and exchange rate pass-through in world automobile trade. Journal of International Economics, 40(1-2), 187-207.

Ganapati, S., Shapiro, J. S., Walker, R., 2017. "The Incidence of Carbon Taxes in U.S. Manufacturing: Lessons from Energy Cost Pass-Through." Unpublished manuscript. 
Gaudin, G., 2016. Pass-through, vertical contracts, and bargains. Economics Letters, $139,1-4$.

Gaudin, G., 2016. Vertical Bargaining and Retail Competition: What Drives Countervailing Power, Economic Journal

Gaudin, G., White, A., 2014. Unit vs. ad valorem taxes under revenue maximization. Manuscript.

Genesove, D., Mullin, W. P., 1998. Testing static oligopoly models: Conduct and cost in the sugar industry, 1890-1914. RAND Journal of Economics, 29(2), 355-377.

Goldberg, P. K., Hellerstein, R., 2013. A structural approach to identifying the sources of local currency price stability. Review of Economic Studies, 80(1), 175-210.

Gopinath, G., Itskhoki, O., Rigobon, R., 2010. Currency choice and exchange rate pass-through. American Economic Review, 100(1), 304-336.

Griffith, R., Nesheim, L., O'Connell, M., 2015. Income effects and the welfare consequences of tax in differentiated product oligopoly. Manuscript.

Häckner, J., Herzing, M., 2016. Welfare effects of taxation in oligopolistic markets. Journal of Economic Theory, 163, 141-166.

Hamilton, S. F., 1999. Tax incidence under oligopoly: a comparison of policy approaches. Journal of Public Economics, 71(2), 233-245.

Hellerstein, R. 2008. Who bears the cost of a change in the exchange rate? Passthrough accounting for the case of beer. Journal of International Economics, 76(1), $14-32$.

Holmes, T. J., 1989. The effects of third-degree price discrimination in oligopoly. American Economic Review, 79(1), 244-250.

Hong, G. H., Li, N. 2017. Market structure and cost pass-through in retail. Review of Economics and Statistics, Forthcoming. 
Johnson, D. B., Pauly, M. V., 1969. Excess burden and the voluntary theory of public finance. Economica, 36(143), 269-276.

Kim, D., Cotterill, R. W., 2008. Cost pass-through in differentiated product markets: The case of U.S. processed cheese. Journal of Industrial Economics, 56(1), 32-48.

Kimmel, S., 1992. Effects of cost changes on oligopolists' profits. Journal of Industrial Economics, 40(4), 441-449.

MacKay, A., Miller, N. H., Remer, M., Sheu, G., 2014. Bias in reduced-form estimates of pass-through. Economics Letters, 123(2), 200-202.

Mahoney, N. and Weyl, E. G., 2017. Imperfect Competition in Selection Markets. Review of Economics and Statistics

Miller, N. H., Osborne, M., Sheu, G., 2017. Pass-through in a concentrated industry: Empirical evidence and regulatory implications. RAND Journal of Economics, 48(1), 69-93.

Miller, N. H., Remer, M., Sheu, G., 2013. Using cost pass-through to calibrate demand. Economics Letters, 118(3), 451-454.

Miller, N. H., Remer, M., Ryan, C., Sheu, G., 2016. Pass-through and the prediction of merger price effects. Journal of Industrial Economics, Forthcoming.

Nevo, A., 1998. Identification of the oligopoly solution concept in a differentiatedproducts industry. Economics Letters, 59(3), 391-395.

Nocke, V., Schutz, N., 2016. Multiproduct-firm oligopoly: An aggregative games approach. Manuscript.

Pigou, A. C., 1928. A study in public finance. Macmillan.

Peitz, M., Reisinger, M., 2014. Indirect taxation in vertical oligopoly. Journal of Industrial Economics, 62(4), 709-755. 
Reny, P. J., Wilkie, S. J., Williams, M. A., 2012. Tax incidence under imperfect competition: Comment. International Journal of Industrial Organization, 30(5), 399-402.

Riordan, M. H., 2008. Competitive effects of vertical integration. In P. Buccirossi (ed.), Handbook of antitrust economics, The MIT Press, 145-182.

Ritz, Robert A., 2017. Oligopolistic competition and welfare. In Luis Corchï¿œn and Marco Marini (eds.), Handbook of Game Theory \& Industrial Organization, Edward Elgar.

Shrestha, V., Markowitz, S., 2016. The pass-through of beer taxes to prices: Evidence from state and federal tax changes. Economic Inquiry, 54(4), 1946-1962.

Skeath, S. E., Trandel, G. A., 1994. A Pareto comparison of ad valorem and unit taxes in noncompetitive environments. Journal of Public Economics, 53(1), 53-71.

Stolper, S. 2016. Who bears the burden of energy taxes? The critical role of passthrough. Manuscript.

Tremblay, M. J., Tremblay, V. J., 2016. Tax incidence and demand convexity in Cournot, Bertrand, and Cournot-Bertrand models. Public Finance Review, Forthcoming.

Vickley, W. W., 1963. General and specific financing of urban services. In H.G. Shaller (ed.), Public expenditure decisions in the urban community, Resources for the Future, 62-90.

Vives, X., 1999. Oligopoly pricing: Old ideas and new tools. The MIT Press.

Weyl, E. G., Fabinger, M., 2013. Pass-through as an economic tool: Principle of incidence under imperfect competition. Journal of Political Economy, 121(3), 528-583.

White, A., Weyl, E. G., 2016. Insulated platform competition. Manuscript.

Yang, J. 1997. Exchange rate pass-through in US manufacturing industries. Review of Economics and Statistics, 79(1), 95-104. 
Zimmerman, P. R., Carlson, J. A., 2010. Competition and cost pass-through in differentiated oligopolies. Manuscript. 